\title{
Analysis and Application of Digital Spectral Warping in Analog and Mixed-Signal Testing
}

\author{
Warwick P. M. Allen, Donald G. Bailey, Member, IEEE, Serge N. Demidenko, Senior Member, IEEE, and \\ Vincenzo Piuri, Fellow, IEEE
}

\begin{abstract}
Spectral warping is a digital signal processing transform which shifts the frequencies contained within a signal along the frequency axis. The Fourier transform coefficients of a warped signal correspond to frequency-domain 'samples' of the original signal which are unevenly spaced along the frequency axis. This property allows the technique to be efficiently used for DSP-based analog and mixed-signal testing. The analysis and application of spectral warping for test signal generation, response analysis, filter design, frequency response evaluation, etc. are discussed in this paper along with examples of the software and hardware implementation.
\end{abstract}

Index Terms-Analog and mixed-signal circuits, chirp, digital signal generation and analysis, electronic testing.

\section{ACRONYMS}

AC Alternate Current

ADC Analog-to-Digital Converter

ALU Arithmetic Logic Unit

ASIC Application-Specific Integrated Circuit

BIST Built-In Self-Test

CMOS Complementary Metal Oxide Semiconductor

DDS Direct Digital Synthesizer

DFT Discrete Fourier Transform

DSP Digital Signal Processing

DUT Device Under Test

ENOB Effective Number of Bits

FFT Fast Fourier Transform

FIR Finite Impulse Response

I/O Input/Output

SW Spectral Warping

\section{NOTATION}

$n, k$

$p, M$

$z, \hat{z}, a, b, c, d$

$j$

$f(t)$

$\left\{f_{n}\right\}$

$\left\{\lambda_{k}(t)\right\}$

$\left\{\varphi_{n}(t)\right\}$

$g_{k}$

$\tilde{g}_{k, n}$

$\psi_{k, n}$

$F[z]$

$F[\hat{z}]$

$m[z]$

$\omega, \omega_{a}, \omega_{b}$

$\hat{\omega}, \hat{\omega}_{a}, \hat{\omega}_{b}$

$\theta(\omega)$

$a_{p}$

$1 / a_{p}^{*}$

$a_{p}^{*}$
$N_{0}, N_{1}, N_{2}$,

$N$

$\hat{N}_{1}, \hat{N}_{2}, \hat{N}_{0 a}$,

$\hat{N}_{\mathrm{Ob}}$,

$n$

$s(\omega)$

$H(\omega)$

$\Phi(\omega)$

$S_{x}(f), S_{y}(f)$

$\sigma_{0}^{2}$

$S_{x}^{*}(f)$ integers

nonnegative integers

complex variables

imaginary unit

continuous time signal

discrete time representation of $f(t)$

set of bases

set of functions

coefficients of the expansion of $f(t)$

coefficients of the expansion of $f_{n}$ coefficients of the expansion of $\left\{\lambda_{k}(t)\right\}$

$z$ transform of $f(t)$

$\hat{z}$-transform of $\left\{f_{k}\right\}$

mapping function between the $z$ and $\hat{z}$ domains

angular frequency in the $z$ plane

angular frequency in the $\hat{z}$ plane

frequency mapping (warping)

zero of the all-pass function

pole of the all-pass function

complex conjugate of $a_{p}$

samples of the sinusoidal input

time corresponding to the samples

time corresponding to the sample $N_{0}$

stretching function

stretching gain response

stretching phase response

frequency spectrum of the signal source

power of the signal source

complex conjugate of $S_{x}(f)$
Manuscript received March 10, 2002. This work was supported in part by Massey University of New Zealand under Massey University Research Fund Grant PR56297.1207 MURF, and Asia 2000 Foundation Grant ED6019.

W. P. M. Allen is currently with the School of EEE, Singapore Polytechnic, Singapore 139651 (e-mail: warwick@sp.edu.sg). From April 2003 he will be with the Institute of Information Sciences and Technology, Massey University, Palmerston North, New Zealand (e-mail: warwick. allen.1@uni.massey.ac.nz).

D. G. Bailey is with the Institute of Information Sciences and Technology, Massey University, Palmerston North, New Zealand (e-mail: d.g.bailey@ massey.ac.nz).

S. N. Demidenko is with the Institute of Information Sciences and Technology, Massey University, Palmerston North, New Zealand. From May 2003 he will be with the School of Engineering and Science, Monash University Malaysia, Kuala Lumpur, Malaysia (e-mail: s.n.demidenko@ieee.org).

V. Piuri is with the Department of Information Technologies, University of Milan, 26013 Crema, Italy (e-mail: piuri@elet.polimi.it).

Digital Object Identifier 10.1109/TR.2003.821933

\section{SUMMARY AND CONCLUSION}

$\mathbf{I}$ N THIS paper, a review and analysis of the Spectral Warping (SW) digital signal processing technique is given, and its application to the area of analog and mixed-signal testing is proposed.

The SW transform shifts the frequency components of a signal along the frequency axis, while maintaining the general shape of the spectrum. However the duration and amplitude of each frequency component are correspondingly affected and have to be taken into account when using the technique.

The proposed new applications of the SW transform are in the emerging area of Digital Signal Processing (DSP)-based 


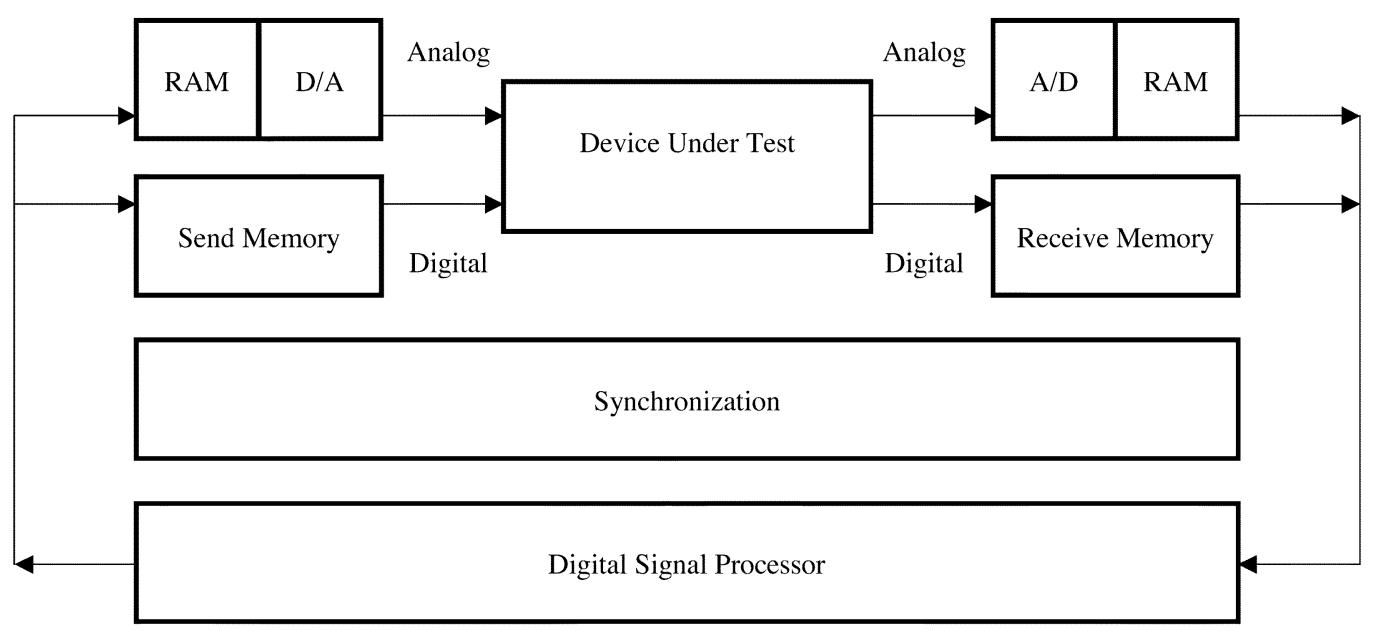

Fig. 1. DSP-based testing of mixed-signal circuit.

analog and mixed-signal testing. They include evaluation of the impulse response of the Device Under Test (DUT) without the risk of overloading and damaging the system, as might happen when applying an impulse. A special chirp signal is generated using SW and applied to the system. The output is fed through two SW networks to produce the impulse response. If only the magnitude response is required, then no SW is needed-simply, the Fourier transform of the chirp response is analyzed. Further, a quick estimate of the magnitude response can be found by observing the envelope of the output. The proposed method is accurate for all but the very extreme frequencies.

Among the other applications of SW in DSP-based electronic testing of analog and mixed-signal systems are the characterization of analog-to-digital converters using input chirp signals, the generation of test signals having pre-specified frequency spectra, digital filtering with good stop-band attenuation, and unequal-resolution frequency analysis.

\section{INTRODUCTION}

The importance of analog and mixed-signal testing has grown increasingly during the last decades. It is no surprise that intensive research and development on the topic has been carried out by many specialists in different countries resulting in a number of techniques and approaches [1]-[8]. Among them, Digital Signal Processing (DSP)-based testing has proved to be one of the most promising, offering excellent potential for practical use. It involves using digital tools and methods to test both digital and analog components [1], [6], [8].

The basic structure of DSP-based testing is presented in Fig. 1. It can be seen that both signal generation and output measurement are realized by means of pure digital circuitry.

Test signals that are usually used in this scheme include [6]-[8]: digitized sinusoid, digitized multi-tone, pseudorandom, etc. The following algorithms are used for response analysis [1], [2], [6]-[8]: Discrete Fourier Transform (DFT) computed by means of Fast Fourier Transform (FFT) [9], IEEE 1057 sine-wave fitting, cross-correlation, auto-correlation, filtering, etc.
DSP-based testing is an effective way around many otherwise unavoidable limits of analog instrumentation (cross-talk, nonlinearity, noise, drift, aging, improper calibration, long filter settling time, thermal effects and so on). At the same time, it provides benefits inherent to the use of digital components and tools (accuracy, stability, single set up for multiple types of tests, results repeatability, etc.). Besides, in DSP-based testing all test access can be obtained through the same digital $I / O$ ports, while the Device Under Test (DUT) can be tested for many parameters in one run thus increasing throughput.

One of the main problems in DSP-based testing of analog and mixed-signal circuits is to find a minimum set of test signals/frequencies and internal test points of the DUT that maximizes the testability [1], [2], [6], [7]. This problem is usually addressed when the circuit is being designed. The other important problem is the generation and application of such test signals to specific points of DUTs as well as analysis of the test responses. These become even more complicated in the Built-In Self-Test (BIST) environment, where available test resources are essentially limited.

This paper introduces a special DSP procedure-Spectral Warping, and discusses how its utilization and implementation can benefit analog and mixed-signal test generation and analysis. The paper is organized as follows. Section II introduces the background theory of Spectral Warping based on the theory first presented in [10], [11]. Properties of the SW transform are investigated in the Section III. Application of Spectral Warping in the Analog and Mixed-Signal test area is discussed in Section IV.

\section{Discrete SPECTRAL WARPING TRANSFORM}

\section{A. Transformation Between Sequences}

Given a continuous time signal $f(t)$, with its discrete time representation $\left\{f_{n}\right\}$, the continuous signal can be represented as the sum of a set of basis functions weighted by a sequence:

$$
f(t)=\sum_{n=-\infty}^{+\infty} f_{n} \varphi_{n}(t) .
$$


Another set of bases which span $f(t)$ may also exist,

$$
f(t)=\sum_{k=-\infty}^{+\infty} g_{k} \lambda_{k}(t)
$$

for which the set of functions $\left\{\varphi_{n}(t)\right\}$ is complete with respect to $\left\{\lambda_{k}(t)\right\}$. Hence each $\lambda_{k}(t)$ can be expanded in terms of $\left\{\varphi_{n}(t)\right\}$ :

$$
\lambda_{k}(t)=\sum_{n=-\infty}^{+\infty} \psi_{k, n} \varphi_{n}(t)
$$

where $\psi_{k, n}$ are the mapping functions between the basis functions, and hence

$$
f_{n}=\sum_{k=-\infty}^{+\infty} g_{k} \psi_{k, n}
$$

For mappings from $f(t)$ to $\left\{f_{n}\right\}$ and from $f(t)$ to $\left\{g_{k}\right\}$ to preserve convolution, the mapping of $\left\{g_{k}\right\}$ to $\left\{f_{n}\right\}$ in (4) also needs to preserve convolution, thus the sequences $\left\{\Psi_{k, n}\right\}$ must fulfill the following condition (see Appendix I for proof):

$$
\Psi_{k}[z]=\left[\Psi_{1}[z]\right]^{k} .
$$

where $\Psi_{k}[z]$ is the $z$-transform of $\left\{\psi_{k, n}\right\}$.

Taking the $z$-transform of (4) we get

$$
F[z]=\sum_{k=-\infty}^{+\infty} g_{k} \Psi_{k}[z]=\sum_{k=-\infty}^{+\infty} g_{k}\left\{\Psi_{1}[z]^{-1}\right\}^{-k}
$$

We will define the $\hat{z}$-transform of $\left\{f_{k}\right\}$ as

$$
F[\hat{z}]=\sum_{k=-\infty}^{+\infty} f_{k} \hat{z}^{-k}
$$

Hence, we can easily see from (6) and (7) that $\hat{z}$ and $z$ are related by the mapping

$$
\hat{z}=\left(\Psi_{1}[z]\right)^{-1} \equiv m[z] .
$$

The mapping from $\left\{f_{n}\right\}$ to $\left\{g_{k}\right\}$ is equivalent to exchanging the complex frequency variable $z$ for $\left\{f_{n}\right\}$ to $\hat{z}$ for $\left\{g_{k}\right\}$. This transformation can be expressed in the $z$ domain as

$$
F[z]=G(m[z]) \text {. }
$$

\section{B. The SW Transform}

A particular set of functions $\left\{\Psi_{k}[z]\right\}$ will result in a transform which creates a new sequence whose Fourier transform is equal to the Fourier transform of the original signal on a distorted (warped) frequency axis [10]. The mapping from one sequence $\left\{f_{n}\right\}$ to another $\left\{g_{k}\right\}$, as expressed by (4), is equivalent to a transformation from $F[z]$ to $G[\hat{z}]$ if (5) is satisfied. If the function $\hat{z}=m[z]$ is defined such that it maps the unit circle in the $z$ plane to the unit circle in the $\hat{z}$ plane, then the transform will be a warping of the frequency axis. If $\omega$ is the angular frequency in the $z$ plane and $\hat{\omega}$ is the angular frequency in the $\hat{z}$ plane, then

$$
e^{j \hat{\omega}}=m\left[e^{j \omega}\right]=e^{j \theta(\omega)}
$$

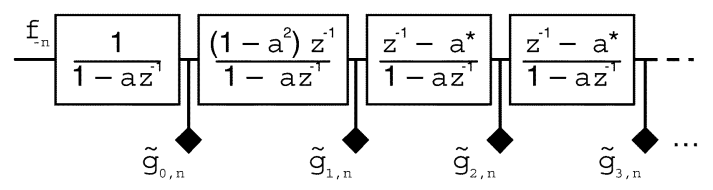

Fig. 2. A spectral warping network.

where $\hat{\omega}=\theta(\omega)$ is the frequency mapping or warping.

These constraints imply that

$$
\left|m\left[e^{j \omega}\right]\right|=\left|\Psi_{1}\left[e^{j \omega}\right]\right|=1
$$

or that $m[z]$ is an all-pass function, i.e.,

$$
\hat{z}=m[z]=\prod_{p} \frac{1-a_{p} z^{-1}}{z^{-1}-a_{p}^{*}}
$$

where $a_{p}$ and $1 / a_{p}^{*}$ are the zeros and poles respectively of the all-pass function, with $a_{p}^{*}$ as the complex conjugate of $a_{p}$.

If the coefficients $a_{p}$ are such that the interval $-\pi<\omega \leq \pi$ maps one-to-one to the interval $-\pi<\hat{\omega} \leq \pi$, then $m[z]$ will be invertible. For this to be true it is necessary (but not sufficient) for the difference between the number of zeros and the number of poles which lie within the unit circle to be one. It is useful to choose a first-order all-pass function, for which (12) reduces to

$$
\hat{z}=m[z]=\frac{1-a z^{-1}}{z^{-1}-a^{*}} .
$$

where $0<|a|<1$ and the subscript 1 has been dropped. The inverse of the above equation, found by replacing $a$ with $-a$, is

$$
z=m^{-1}[\hat{z}]=\frac{1+a \hat{z}^{-1}}{\hat{z}^{-1}+a^{*}} .
$$

The set of $z$-transforms $\left\{\Psi_{k}[z]\right\}$ which relate $f_{n}$ and $g_{k}$ is

$$
\Psi_{k}[z]=\left(\frac{z^{-1}-a^{*}}{1-a z^{-1}}\right)^{k}
$$

Thus $\Psi_{k}[z]$ corresponds to a $k$ th-order all-pass filter with $k$ poles at $z=a$ and $k$ zeros at $z=1 / a^{*}$.

\section{Realizing the SW Transform}

A SW network is an all-pass filter of the form shown in (15) and can be realized as a cascade of first-order all-pass filters. A signal is time reversed and passed through the first all-pass filter. The last sample of the filtered sequence becomes the first output sample. The sequence is then passed through another all-pass filter. The last sample of the sequence after the second filter is the second output sample. This process is repeated for each filter in the network. The number of output samples generated is equal to the number of filters used. Fig. 2 shows the form of a SW network. All of the filters are identical apart from the first two [11].

Fig. 3 illustrates how a signal is shifted in frequency by means of the SW network. A sequence representing a sinusoid of $\pi / 4$ ( $\pi$ is half the sampling rate) is taken. A SW network with the warping factor $a$ is chosen such that a phase shift of $\pi / 16$ is experienced at $\omega=\pi / 4$. Fig. 3(a) shows the sequence after it has been time-reversed and fed through the first filter, and the last sample is taken as the first output sample. Fig. 3(b) presents how the output sequence is formed after the signal has been 


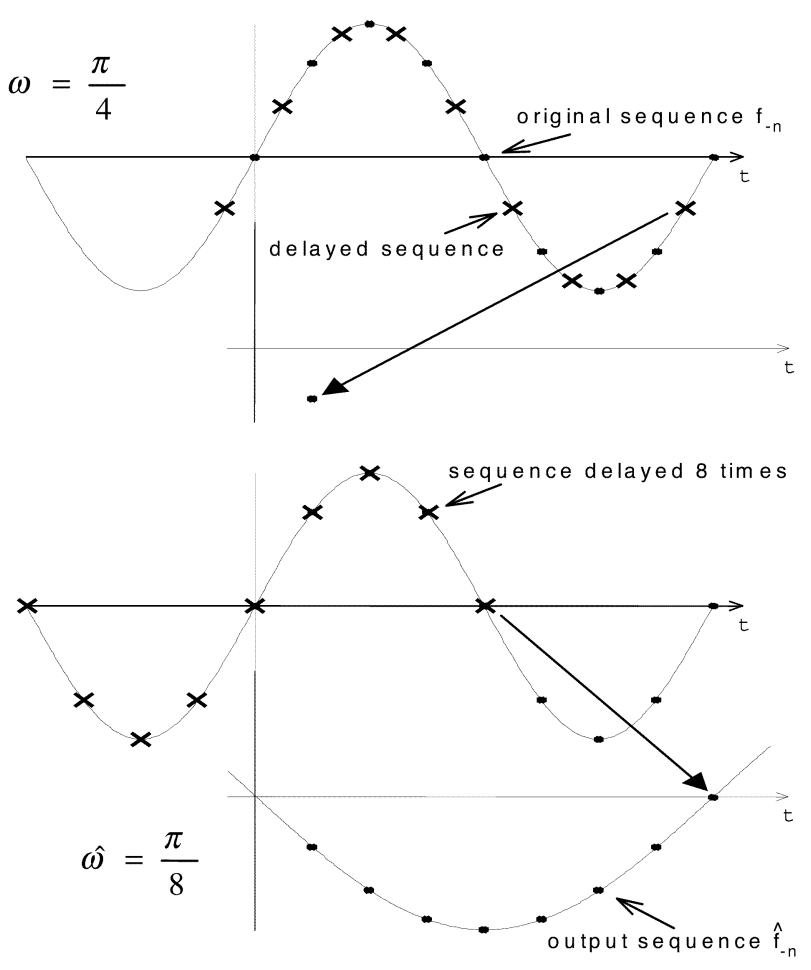

Fig. 3. Frequency shift realization by the SW all-pass network.

through several filters. In this example, the output frequency is half that of the input.

\section{ANALYSIS OF SW PROPERTIES}

\section{A. Frequency Distortion}

The frequency components of a signal that has undergone SW are shifted in the frequency domain. This distortion of the frequency axis is discussed in [10], [11]. The extent of the distortion, controlled by the parameter $a$ (called the warping factor), is given by

$$
\begin{aligned}
\hat{\omega} & =\theta(\omega) \\
& =\tan ^{-1}\left[\frac{\left(1-a^{2}\right) \sin \omega}{\left(1+a^{2}\right) \cos \omega-2 a}\right] \quad-1<a<1,
\end{aligned}
$$

and the inverse relationship is

$$
\begin{aligned}
\omega & =\theta^{-1}(\hat{\omega}) \\
& =\tan ^{-1}\left[\frac{\left(1-a^{2}\right) \sin \hat{\omega}}{\left(1+a^{2}\right) \cos \hat{\omega}+2 a}\right] \quad-1<a<1 .
\end{aligned}
$$

Distortion curves for a selection of warping factors are given in Fig. 4. It can be seen that the closer the $|a|$ is to one, the more severe the distortion. The symmetry between the curves for a particular warping factor and the negative of that warping factor shows that the transform is reversible. A signal produced by warping with a factor of $a$ can be transformed back to its original form by warping with $-a$. A complex-valued warping factor enables the point of maximum distortion to be positioned at any frequency.

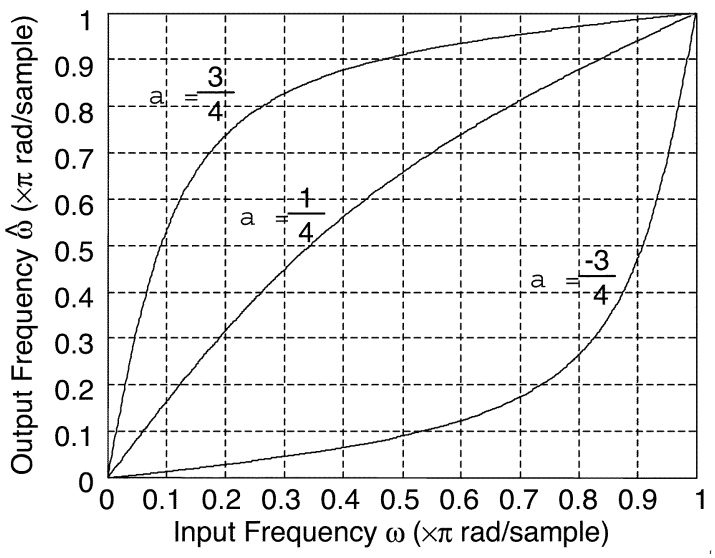

Fig. 4. Frequency mapping curves.

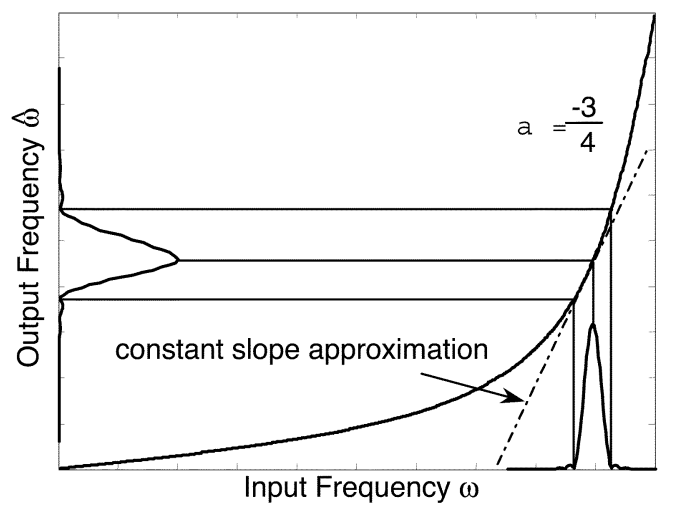

Fig. 5. Distortion of a signal's bandwidth.

\section{B. Time Distortion}

To transform a segment of a signal that extends for all time, the signal is multiplied by a windowing function. This corresponds to convolving the spectrum of the signal with that of the window. If the bandwidth of the signal is much narrower than the bandwidth of the windowing function, then the signal's spectrum takes on the shape of the spectrum of the windowing function, but remains at the same center frequency. When the windowed signal is transformed this spectrum is warped according to the slope of (16). If the bandwidth of the windowing function is sufficiently narrow, this slope can be approximated to be constant (as illustrated in Fig. 5).

There is a reciprocal relationship between the time and frequency domains. This means that the duration of the signal is reduced if the bandwidth is increased, and vice versa. Therefore the change in duration, or stretching, given below in (18), is the reciprocal of the derivative of (16).

$$
\begin{aligned}
s(\omega) & =\frac{1}{\frac{d \theta(\omega)}{d \omega}} \\
& =\frac{\left(1-2 a \cos \omega+a^{2}\right)}{\left(1-a^{2}\right)} .
\end{aligned}
$$

A sinusoidal input of frequency $\omega$, starting at sample $N_{1}$ and finishing at $N_{2}$, will produce a sinusoidal output of frequency 


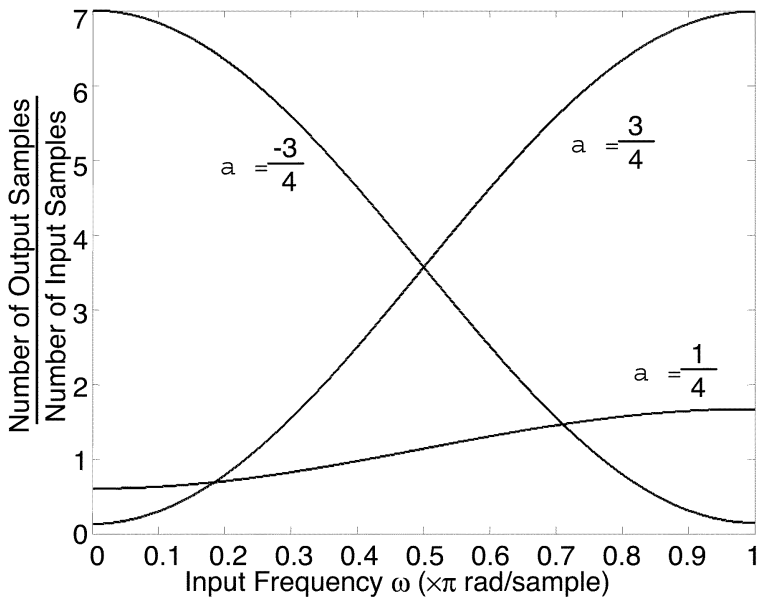

Fig. 6. Ratio of the duration of the output to that of the input as a function of the input frequency for three warping factors. For a warping factor of $\pm 3 / 4$, seven times the number of output samples as input samples are needed to completely represent the warped version of a signal containing all possible frequencies.

$\hat{\omega}=\theta(\omega)$, spanning the time $\hat{N}_{1}$ to $\hat{N}_{2}$. Substituting $N_{1}$ and $N_{2}$ for $N$ in (19) will give these values.

$$
\begin{aligned}
\hat{N}(N, \omega) & =N \times \frac{\left(1-2 a \cos \omega+a^{2}\right)}{\left(1-a^{2}\right)} \\
& =N s(\omega) .
\end{aligned}
$$

A result of this time distortion is the number of samples needed to completely represent the output signal for an arbitrary input is the length of the input signal multiplied by either $s(0)$ or $s(\pi)$, depending on the sign of $a$. An example is shown in Fig. 6.

\section{Amplitude and Phase Distortions}

The energy of each frequency component is conserved during a SW transformation (providing there are enough output samples to ensure that the signal is not truncated). Therefore when a frequency component is stretched out in time it is also attenuated. Similarly, it is amplified if it is compressed in time. The expression for the magnitude gain response is therefore the derivative of (16):

$$
|H(\omega)|=\frac{\left(1-a^{2}\right)}{\left(1-2 a \cos \omega+a^{2}\right)} .
$$

This result was originally derived in [12], along with the phase response, which is shown below.

$$
\Phi(\omega)=(1-M) \tan ^{-1}\left[\frac{\left(1-a^{2}\right) \sin \omega}{\left(1+a^{2}\right) \cos \omega-2 a}\right]
$$

where $M$ is the number of filters.

The amplitude distortion, being a function of both frequency and $a$, gives rise to the three-dimensional bode plot of Fig. 7 .

\section{SW Applications in Circuit Testing}

\section{A. Unequal-Frequency Resolution Signal Generation and Analysis}

The spectral warping technique (originally developed for speech correction) can find new, interesting and efficient applications in the emerging area of analog and mixed-signal

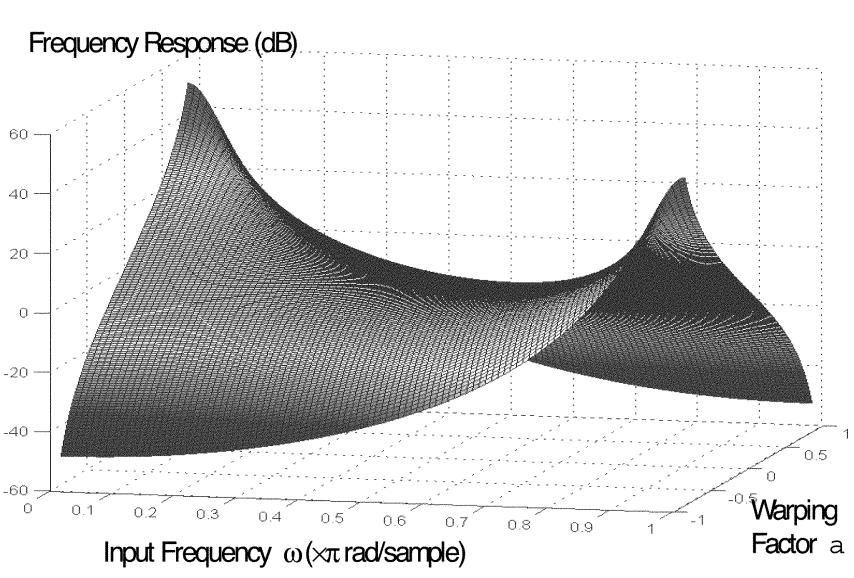

Fig. 7. Frequency response of a SW network.

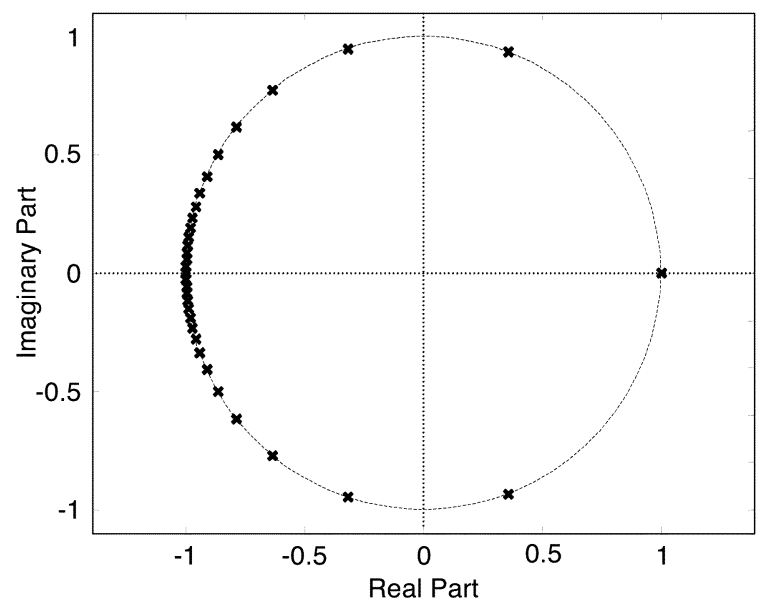

Fig. 8. Example of the samples in the $z$-space found by first spectrally warping a signal and then taking a Fourier transform.

testing when unequal frequency resolution is required. This offers significant benefits in mass-production testing where high throughput of testing is vitally important. The use of frequency spectral warping pre-processing to obtain unequal spectral resolution could reduce total computing times and thus time to perform testing. This could be especially important in testing high-speed high-frequency circuits and systems.

Indeed, SW enables the Fast Fourier Transform (FFT) to have unequal resolution along the frequency axis. This relates to taking samples of the $z$-transform that are unevenly distributed around the unit circle, as shown in Fig. 8.

A signal can be pre-warped to move frequency components away from a band of particular interest. The results of an FFT of the warped signal are shifted back in frequency by applying the inverse of (16). The data now reflect the frequency content of the unwarped signal, with the frequency data being closely spaced where the slope of $\theta(\omega)$ is the steepest. This technique is most useful in the case when information from one end of the spectrum is important, while the other end of the spectrum can be ignored.

For example, if it were desired to analyze a signal's frequency content above $0.9 \pi$ (and the information about the rest of the spectrum is of no interest), then the signal could be prewarped using $a=-0.8$. An output sequence of $M=N / 2$ could be 


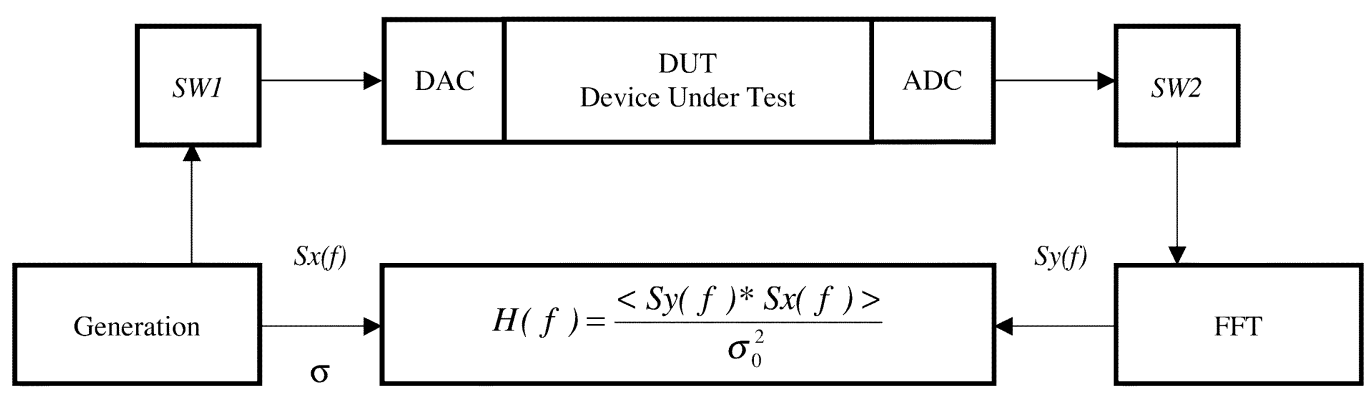

Fig. 9. SW-based testing arrangement.

used as it would not matter that the lower frequency signal components would be truncated. The FFT of the warped signal could then be generated and the result plotted against

$$
\bar{\omega}=\theta^{-1}(\hat{\omega})=\tan ^{-1}\left[\frac{\left(1-a^{2}\right) \sin \hat{\omega}}{\left(1+a^{2}\right) \cos \widehat{\omega}+2 a}\right]
$$

where $\hat{\omega}=\theta(\omega)$. So if the frequencies of interest lay in this upper band, then an FFT could be performed on a sequence of half the length, yielding higher resolution while performing fewer calculations.

It is important that SW can be employed for both test response analysis and test signal generation when nonuniform frequency resolution ("focused" or "stretched") is required. This is of particular importance in telecommunication or audio/video circuits and systems testing. The test data flow is shown in Fig. 9. In both the cases (test stimuli generation and response analysis) the amplitude-frequency and phase-frequency characteristics of the network, as presented in Section III, have to be taken into account to obtain correct results from testing.

A signal is applied to the DUT from a signal source of known frequency spectrum $S_{x}(f)$ and power $\sigma_{0}^{2}$. The signal output from the DUT is converted from analog to digital form and transformed by FFT into frequency spectra $S_{y}(f)$. To limit the measurement errors caused by internal noise, ensemble averaging can be used, and the transfer function, $H(f)$, is found as

$$
H(f)=\frac{\left\langle S_{y}(f) S_{x}^{*}(f)\right\rangle}{\sigma_{0}^{2}}
$$

where \langle\rangle denotes ensemble averaging and $S_{x}^{*}(f)$ is the complex conjugate of $S_{x}(f)$.

SW is used for both the generation of unequally spaced frequencies (with some value of warping parameter $a$ ), and for the response analysis (with the warping parameter equal to $-a$ as the frequencies have to be placed back into the uniform mode). Thus the total distortion introduced by the double warping is

$$
\left|H_{M}(\omega)\right|=\frac{\left(1-a^{2}\right)^{2}}{\left(1+a^{2}\right)^{2}-4 a^{2} \cos ^{2} \omega}
$$

To eliminate the amplitude distortion an appropriate correction has to be realized. It can be done, for example, by means of a digital filter having amplitude-frequency characteristics inverse to $H_{M}(\omega)$.

\section{B. Using SW Chirps to Find Systems' Frequency Response}

Another way of testing analog and mixed-signal circuits is based on the evaluation of the frequency response of a DUT. To find the frequency response of a system, one can apply an impulse to the input and measure the response on the output. To provide good accuracy of the measurement, the generated impulse has to be of substantial energy.

However, because all the energy in an impulse signal is concentrated in one point in time (ideally, the pulse has to be unlimitedly short and with unlimitedly high magnitude), this method can damage delicate electronic circuitry. Spectral warping allows this situation to be avoided by converting an initial pulse into an equivalent chirp signal. Such a signal contains frequencies from the entire spectrum, so the frequency response can also be derived from its chirp response.

An impulse at sample $N_{0}$ can be constructed with a linear combination of cosine functions that add at $n=N_{0}$ and cancel for $n \neq N_{0}$.

Because superposition holds for the SW transform, we can analyze the transformation of an impulse by observing the transform of the sinusoids that make it up. It is useful to consider the sinusoidal components as spanning the whole sequence, i.e., from $n=N_{1}=0$ to $n=N_{2}=N-1$. Each transformed component still begins at the origin $\left(\hat{N}_{1}=0 s(\omega)\right)$, but lasts until $\hat{N}_{2}=N_{2} s(\omega)$. The phase of each component at the origin is also unchanged.

The sinusoids no longer add together at one point and cancel each other at all the other points. However, components of similar frequency do, to some extent, constructively interfere at a certain time and destructively interfere at other times. If two components of frequencies $\omega_{a}$ and $\omega_{b}$ are transformed to the frequencies $\hat{\omega}_{a}$ and $\hat{\omega}_{b}$, the point where they originally coincided, $N_{0}$, is transformed to $\hat{N}_{0 a}$ and $\hat{N}_{0 b}$, respectively. If, $\omega_{a} \approx \omega_{b}$ then $\hat{N}_{0 a} \approx \hat{N}_{0 b}$ and constructive interference will occur at that time. So if an impulse is transformed, the time in the output sequence at which each frequency will have its maximum power is given by (19), substituting $N_{0}$ for $N$. The result is a signal in which the low frequencies have a maximum at one end and the high frequencies have a maximum at the other. The time at 


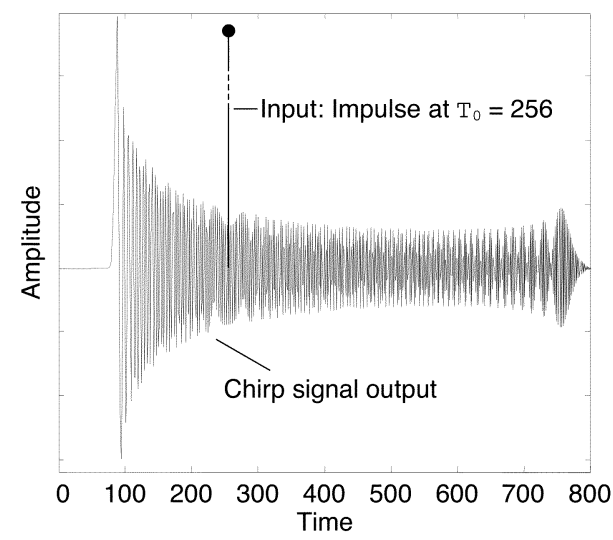

Fig. 10. Chirp signal generated by warping an impulse (SW factor $a=0.5$ ).

which each frequency has its maximum is found by replacing $\omega$ with its expression in terms of the distorted frequency from (17).

$$
\hat{N}_{0}(\hat{\omega})=N_{0} \frac{1-a^{2}}{1+a^{2}+2 a \cos \hat{\omega}}
$$

Fig. 10 shows a chirp signal generated by SW. The warping factor is positive, so the chirp begins with low frequencies and finishes with high frequencies. It can be seen that beating appears toward the tail of the chirp signal. By this time only the high-frequency components are present. The few remaining frequency components move in and out of phase, causing constructive and then destructive interference, resulting in beating.

Up to a certain point all the frequency components are present in the output signal and they cancel each other out. The chirp signal begins when the first frequency component finishes. This component will be the one relating to either $\omega=0$ or $\omega=$ $\pi$, depending on the sign of $a$. If the appropriate value of $\omega$ is substituted into (19), the start of the chirp, $N_{1}$, can be found as a function of the time of the impulse.

$$
N_{1}=N_{0} \times \frac{(1-|a|)^{2}}{\left(1-a^{2}\right)} .
$$

The end of the chirp is found in a similar manner:

$$
N_{2}=N_{0} \times \frac{(1+|a|)^{2}}{\left(1-a^{2}\right)} .
$$

To find a system's frequency response, a test chirp is generated by spectrally warping an impulse, using a warping factor $a$. This signal is then passed through the system or circuit to be tested. This gives the system's chirp response.

The Fourier transform of the chirp response has the same magnitude as the system's frequency response. This is because each frequency component of the chirp has equal energy. The phase information is altered due to the time shifting.

To recover the phase information the chirp response is warped with $-a$. This produces a warped impulse response that is time-shifted by $N_{0}$. Shifting this to start at the origin and then warping it (using the original warping factor $a$ ) will make it identical to an impulse response found in the conventional

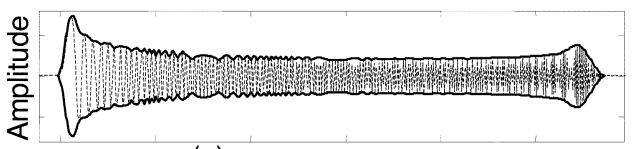

(a)

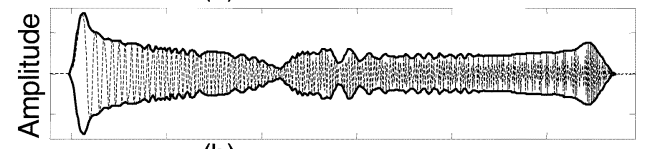

(b)

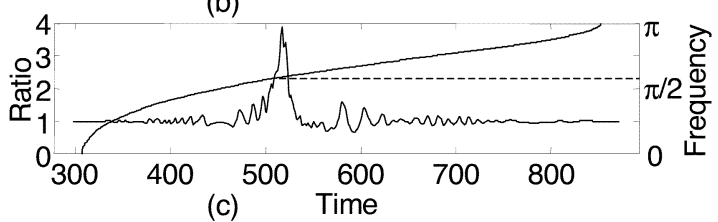

Fig. 11. Envelopes of a chirp signal (a) and the chirp response (b) of a band-stop filter along with the ratio of the two (c) overlaid with the frequency versus time curve.

manner. The system's frequency response can be found from the Fourier transform of the impulse response.

It is possible to estimate a system's magnitude response from its chirp response without calculating a Fourier transform. Because the time at which each frequency is dominant in the chirp is known from (25), an indication of the magnitude response can be obtained by comparing the envelope of the chirp response with that of the chirp signal. Fig. 11 shows an example of a chirp response of a band-stop filter with a very narrow stop-band. Only one section of the chirp response's envelope differs significantly from the envelope of the original chirp. This occurs at the time when the stop-band frequency is dominant in the chirp. This frequency can be found by overlaying the frequency versus time curve for the particular chirp. (The known stop band for this example filter is $0.57 \pi$ to $0.58 \pi$ ). This approximation is only valid if the phase shift introduced by the system is not excessive.

In addition to the evaluation of the frequency response function, SW can benefit testing and characterization of other parameters of mixed-signal systems. For example, it can accelerate evaluating the dependence between an Effective Number of Bits (ENOB) of Analog-to-Digital Converter (ADC) and the input signal frequency. The basic idea of this approach is to bring a full-swing chirp signal to input of the tested ADC where the frequency sweep of the chirp covers the required range of the dynamic test, while the length of the chirp is determined by the required accuracy of the test. The estimation of ENOB with respect to the input signal frequency is then calculated from the series of ADC output samples using an algorithm developed in [13], [14].

The approach provides the same accuracy as a sine-fit test (one of the basic methods for ADC testing as specified by IEEE Std 1241-2000 [15]). At the same time it requires shorter test time that is a very valuable advantage in ADC final production testing.

It was pointed out in [14] that a superior chirp generator is required to implement the method. This is due to the fact that most Direct Digital Synthesizer (DDS)-based generators are not suitable for such an application as their frequency sweep is built 


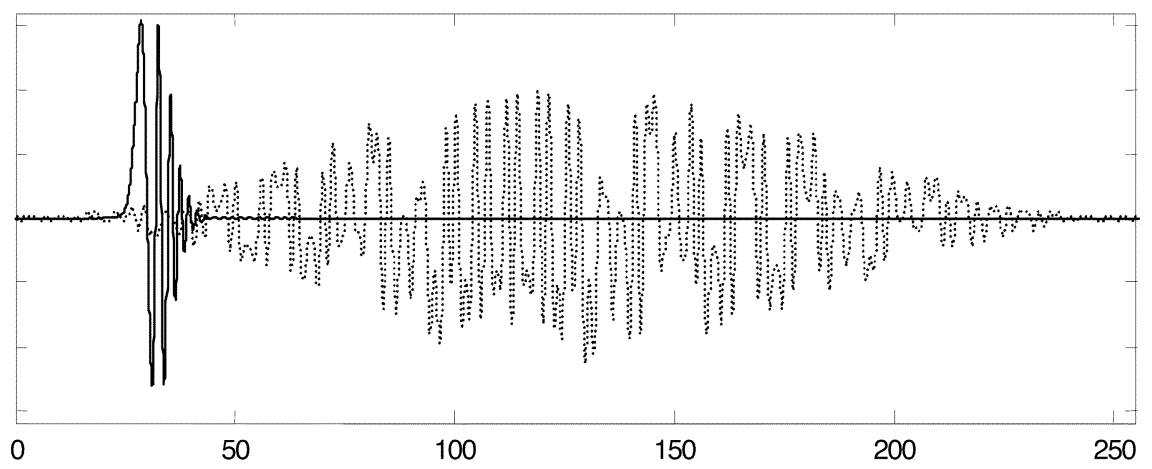

Fig. 12. Spectral warping (solid line) and linear (dotted line) chirp signals.

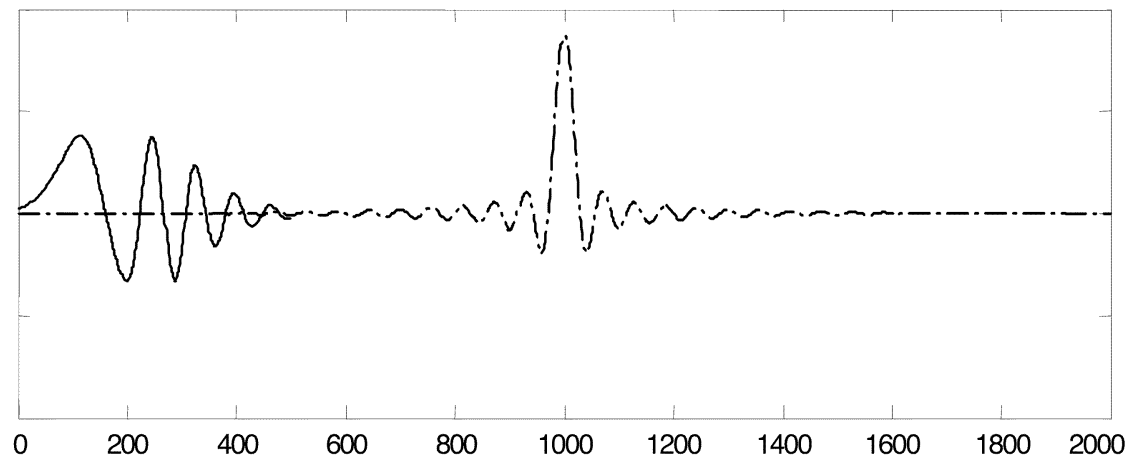

Fig. 13. Comparison between a SW chirp (solid line) and a Parks-McClellan (broken line) low-pass filter.

upon discrete frequency incrementing thus degrading the test results. The required chirp generator can be implemented employing SW-based technique providing required both frequency and amplitude parameters of the test signal.

\section{Using SW for Test/Response Signal Filtering}

Digital filtering is one of the most widely used procedures in DSP-based testing. Among the main requirements of the filter employed in test generation/response analysis are simplicity, speed and stability. This is particularly important in the BIST environment where allowed test overhead is often very limited.

Finite-impulse-response (FIR) filters generally satisfy the above requirements, and thus they are widely used in test generation/analysis [8]. SW provides a new efficient way of designing FIR filters. A SW chirp can be upsampled and used as the filter coefficients for a low-pass FIR filter. The low-pass filter can be transformed to a high-pass, band-pass or band-stop filter using appropriate spectral transformation techniques [16]. The process of developing a low-pass filter using the SW procedure is as follows.

A SW chirp is produced using a warping factor $a=1 / 16$. This chirp is shown in Fig. 12 along with a linear chirp (multiplied by a Hanning window function [9]) for comparison.

The chirp signals are then up-sampled by a factor of 32 . This produces the filter coefficients. Because the SW chirp is (almost) zero for most of its length, the leading and trailing zero coefficients are removed. In this case, the 501 coefficients re- main. The linear chirp filter retains a length of 1892 . The SW chirp filter is plotted in Fig. 13. Sharing the same axes is a plot of a Parks-McClellan FIR filter, designed to have the same characteristics as the two chirp filters.

Fig. 14 shows the frequency response of the three filters (SW chirp filter, linear chirp filter and Parks-McClellan filter). It appears from Fig. 14 that the SW chirp filter can achieve a comparable magnitude response to a Parks-McClellan filter of four times the order. A SW chirp filter will give a much better magnitude response than a Parks-McClellan filter of the same order.

The linear chirp filter has much more ripple in the pass-band and requires a much larger order to achieve similar stop-band characteristics as the SW chirp filter.

It has to be mentioned, however, that the SW method produces a much greater phase shift. A related disadvantage is that the SW chirp filter produces a large, nonconstant group delay, as illustrated in Fig. 15.

Summarizing, the SW chirp filter provides significant advantages where fast (it can be a smaller order than ParksMcClellan), simple (FIR filters are simpler to implement than IIR ones) and stable (FIR filters are always stable) filtering is required.

\section{EXPERIMENTAL RESULTS}

Applicability and efficiency of the SW technique in DSPbased testing was verified experimentally. To evaluate the ability 


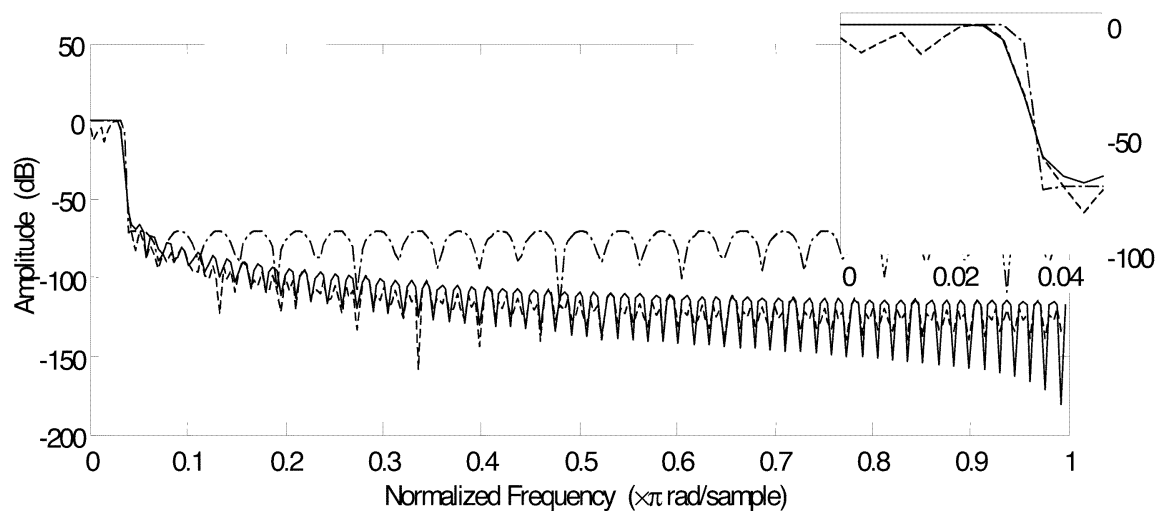

Fig. 14. Magnitude frequency response of the SW chirp (solid) filter compared with the linear chirp (dotted) and the Parks-McClellan (broken) filters.

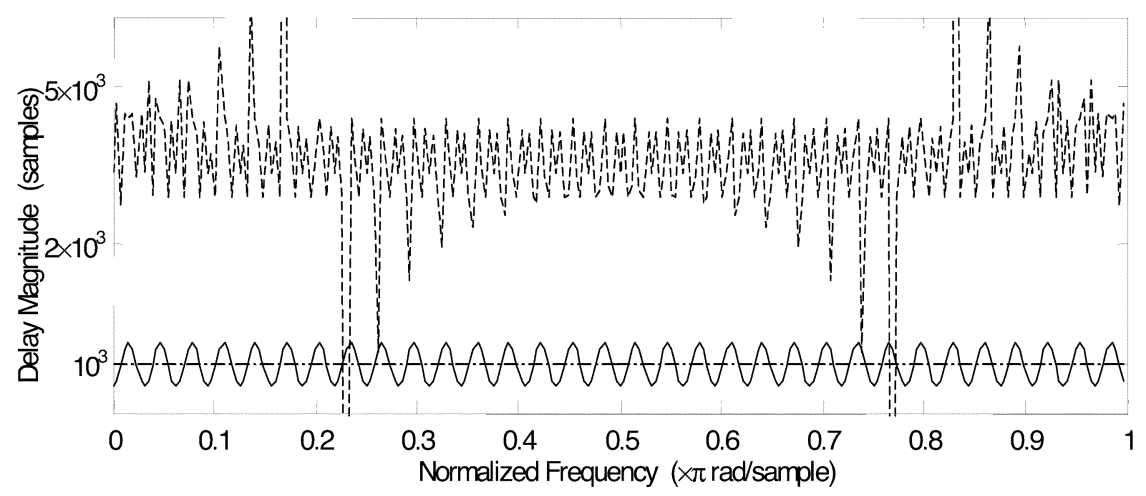

Fig. 15. Group delay of the SW chirp (solid), linear chirp (dotted) and Parks-McClellan (broken) filters.

and accuracy of the SW technique to find a circuit's response, two simple filters were used as DUTs.

\section{A. Experiment Set-Up}

The first simulation arrangement was done using MATLAB. A software implementation of the SW network (the algorithm is presented in Fig. 16) used MATLAB's filter function to directly implement the spectral warping network (Fig. 2). A test chirp was generated using the SW network and fed into the DUT (a band-pass filter). The chirp response was analyzed according to the method described in Section IV-B

In the second experimental set-up, a MATLAB-generated SW chirp signal was used as the input to a filter designed employing PSPICE. The simulation was performed using PSPICE and the simulation output was loaded back into MATLAB for processing. An AC sweep analysis was performed using PSPICE, providing data for comparison. The DUT was an active high-frequency boost filter with a maximum gain of two, using an LM324 Op-Amp as the active component. The netlist for the circuit is found in Appendix II.

\section{B. Results}

For the first experiment, the $\mathrm{SW}$ technique produced a result identical to the known response of the MATLAB filter DUT (ex-

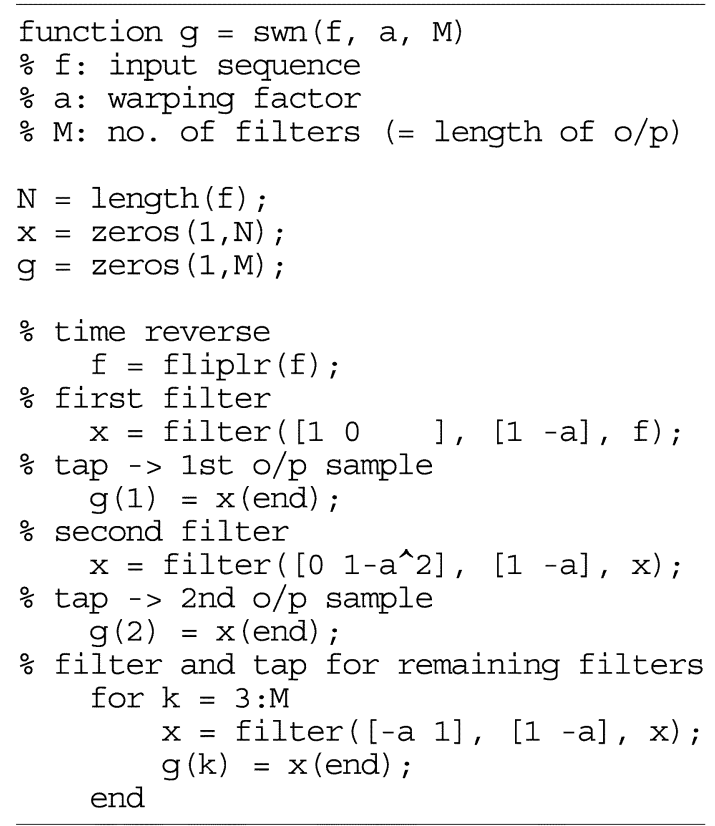

Fig. 16. MATLAB implementation of a SW network. 

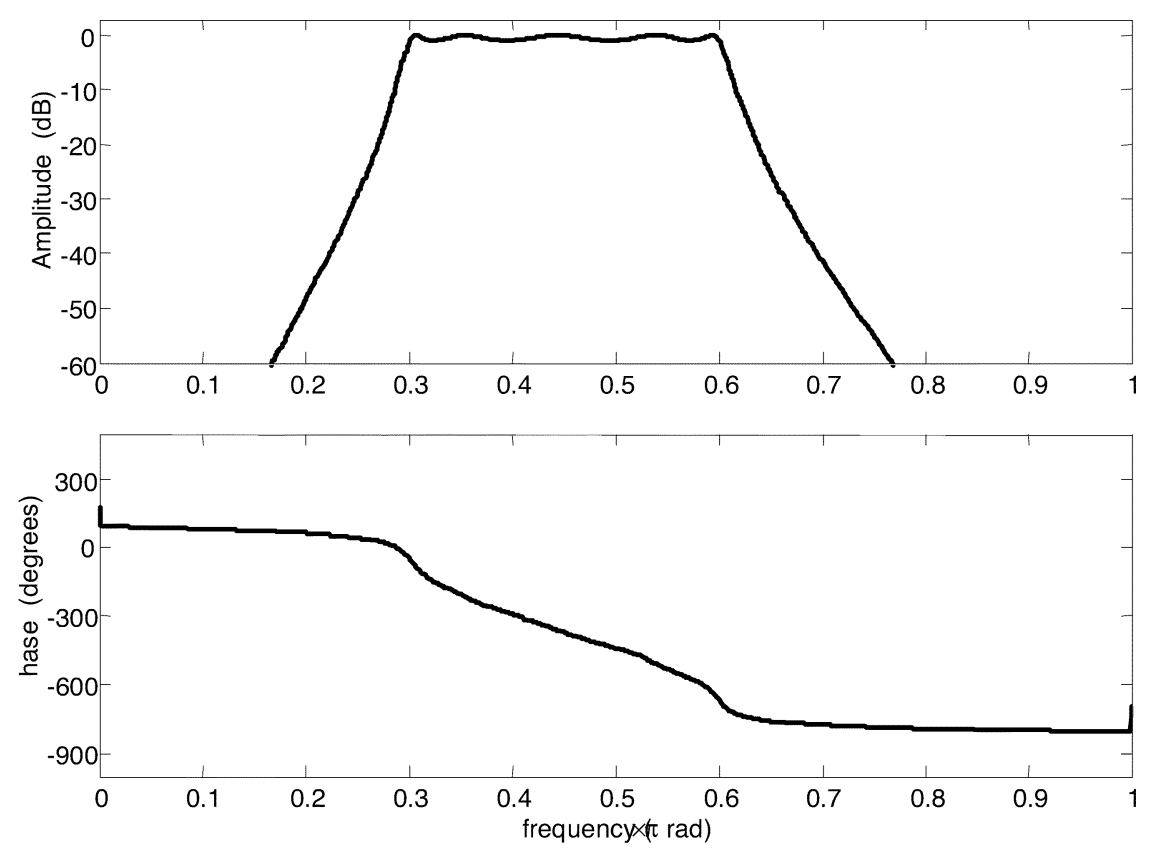

Fig. 17. Band-pass filter used in Matlab to test SW chirp system technique. There was no visible difference between the actual and the calculated frequency response.
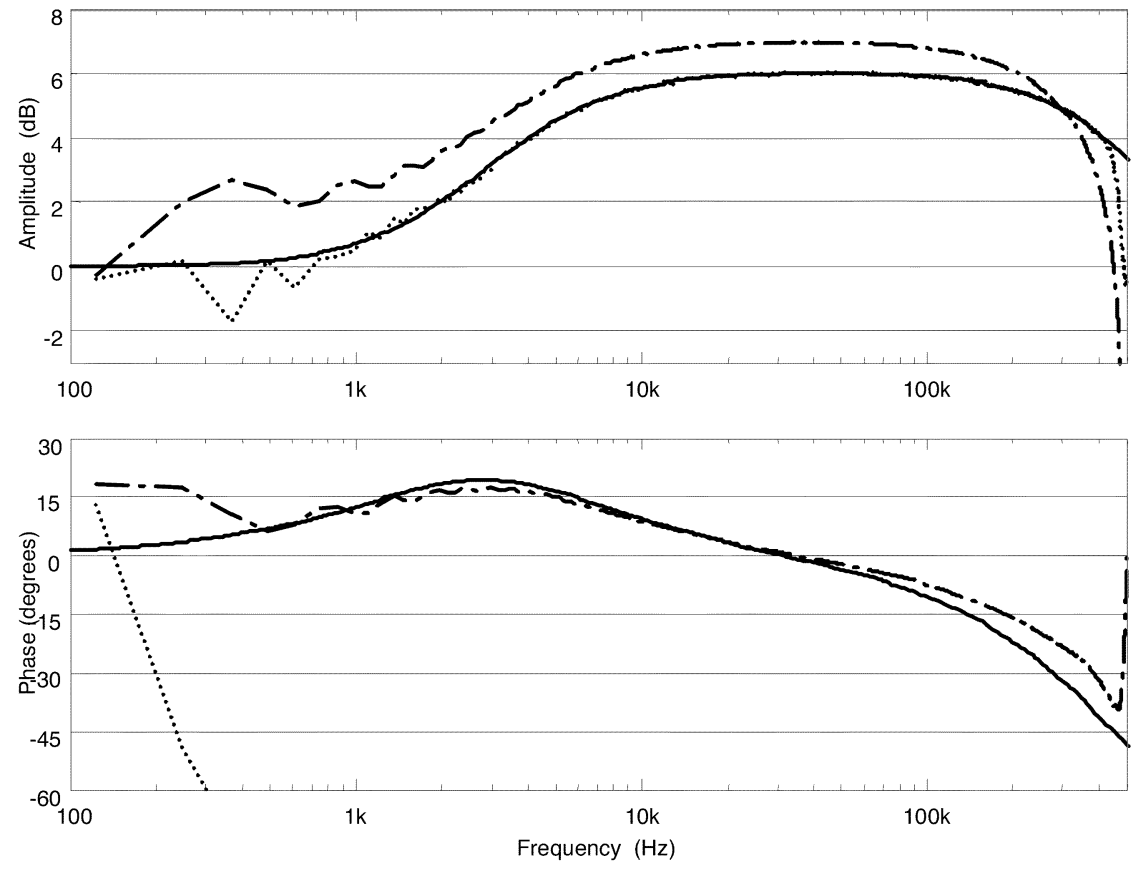

Fig. 18. Frequency response of PSPICE simulated filter. The solid line is from the PSPICE AC sweep analysis, the dotted line is the Fourier transform of the SW chirp response before phase adjusting and the broken line is the Fourier transform of the SW chirp response after phase adjusting.

cept for the very first and the very last samples). The response is shown in Fig. 17.

When comparing the results from the PSPICE simulation, the magnitude of the Fourier transform of the SW chirp response is very close to the magnitude part of the PSPICE AC sweep analysis, deviating only at the extremely high and low frequencies (see Fig. 18). After correcting for the phase, the phase response agrees with that of the AC sweep within a few degrees for $0.001 \pi<\omega<0.2 \pi$ and within $8^{\circ}$ up to $0.93 \pi$. The magnitude becomes less accurate (in this case gaining about $1 \mathrm{~dB}$ over most of the spectrum) after the phase correction. 


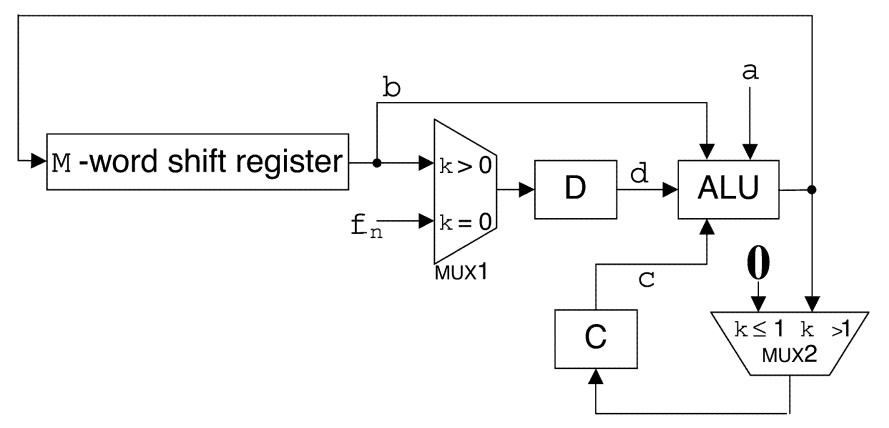

Fig. 19. Hardware implementation of the SW transform.

\section{IMPLEMENTATION OF A SW NETWORK}

Evaluation of practicability of the employing SW in electronic testing (in particular, in the BIST environment) would require development and analysis of its corresponding microelectronic implementation.

An initial architectural hardware solution (Fig. 19) was proposed in [10], [11]. This architecture is derived from representing the filter network of Fig. 2 with the set of difference equations:

$$
\begin{aligned}
\tilde{g}_{0, n} & =a\left[\tilde{g}_{0, n-1}-0\right]+f_{n} \\
\tilde{g}_{1, n} & =a\left[\tilde{g}_{1, n-1}-0\right]+\tilde{g}_{0, n-1} \\
\tilde{g}_{k, n} & =a\left[\tilde{g}_{k, n-1}-\tilde{g}_{k-1, n}\right]+\tilde{g}_{k-1, n-1} \\
k & =2,3, \ldots .
\end{aligned}
$$

By defining the following variables

$$
\begin{aligned}
& a=\text { warping factor (constant) } \\
& b=\tilde{g}_{k, n-1} \\
& c= \begin{cases}\tilde{g}_{k-1, n} & n>0 \\
0 & n=0\end{cases} \\
& d= \begin{cases}\tilde{g}_{k-1, n-1} & k>0 \\
f_{n-1} & k=0\end{cases}
\end{aligned}
$$

and the function

$$
F[a, b, c, d]=a(b-c)+d,
$$

the equations of (28) can be rearranged to

$$
\tilde{g}_{k, n}=F[a, b, c, d]
$$

giving

$$
\begin{aligned}
& \tilde{g}_{0, n}=F\left[a, \tilde{g}_{0, n-1}, 0, f_{-n}\right] \\
& \tilde{g}_{1, n}=F\left[a, \tilde{g}_{1, n-1}, 0, \tilde{g}_{0, n-1}\right] \\
& \tilde{g}_{k, n}=F\left[a, \tilde{g}_{k, n-1}, \tilde{g}_{k-1, n}, \tilde{g}_{0, n-1}\right] \quad 0 \leq k<M .
\end{aligned}
$$

\begin{tabular}{|c|c|c|c|}
\hline Stage & Input & Operation & Output \\
\hline 1 & $c_{1}$ & $-c_{l}$ & 0 \\
\hline 2 & $b_{1}$ & $b_{l}-c_{1}$ & 0 \\
\hline 3 & $d_{l}$ & $a\left(b_{l}-c_{l}\right)+d$ & 0 \\
\hline 1 & $c_{2}$ & $-c_{2}$ & $a\left(b_{1}-c_{1}\right)+$ \\
\hline 2 & $b_{2}$ & $b_{2}-c_{2}$ & $a\left(b_{1}-c_{1}\right)+$ \\
\hline 3 & $d_{2}$ & $a\left(b_{2}-c_{2}\right)+d_{2}$ & $a\left(b_{1}-c_{1}\right)+$ \\
\hline & & : & \\
\hline
\end{tabular}

For each (time-reversed) input which is fed into MUX1, $M$ calculations are performed ( $M$ is the number of filters) with the results being shifted into the shift register. Once the entire input sequence has been fed through the system, the shift register contains the output sequence. A core element of this hardware implementation is the special-function arithmetic logic unit (ALU) that calculates (30).

To evaluate hardware overhead and speed of operation an ASIC to calculate (30) has been designed and simulated for 5-bit
TABLE I

The Stages of Data LOADING and Calculating

word lengths (a compromise between design complexity and resolution). The chosen target technology was $0.6 \mu \mathrm{m}$ triplemetal single-poly CMOS from AMI Semiconductors Inc. With the aim of real integrated circuit implementation the design was also converted into more advanced $0.18 \mu \mathrm{m} 5$ metal CMOS technology. Several different implementation architectures were used (sequential, combinational, and mixed).

The dataflow diagram for the sequential architecture is presented in Fig. 20 [17]. It reads all the input words sequentially from the same I/O pads. It can evaluate $F 8.33$ million times a second running from a $25 \mathrm{MHz}$ clock, and occupies $1.52 \mathrm{~mm}^{2}$. The number of I/O pads is $14(4 \times 4$ square with two unused). The chip accepts five-bit signed magnitude binary input words and the output is presented in the same form. If an overflow has occurred the output is set to \pm 15 . An overflow output bit, which indicates whether an overflow has occurred at any stage of the calculation, is also provided. No overflow occurs during the multiplication stage of the calculation because the product is truncated. The four high-order bits of the product are used, while the low-order bits are discarded.

The design takes advantage of the fact that identical operations are performed on the data at different points in the calculation. This means that circuitry can be reused within the calculation. To do this, intermediate results are stored in a register. The four input words $(a, b, c$ and $d)$ are loaded at separate times. This significantly reduces the pin-count and, hence, the chip-size (the silicon space is normally a limiting factor in the design). However, because such a solution slows the processing, if the operation speed is imperative, then parallel loading of the input values has to be implemented.

Because $a$ is a constant in the spectral warping transform, it is loaded into a register at the start of the transform, and the data in that register is reused for the subsequent iterations of the function. One pad (LDA) is provided to indicate that the data on the input pads is to be loaded into the $a$ register on the next rising edge of the clock. The LDA signal also acts as a reset, clearing the output register and resetting the chip back to the first stage of the calculations. As a result of the truncation in the multiplication stage, $a$ is inherently scaled by $2^{-4}$. To cancel this effect $a$ should be pre-scaled by $2^{4}$. This is useful for the spectral warping, where $-1<a<1$. The stages of ALU operation are listed in Table I.

Besides the ALU, the most silicon space-consuming element of the SW architecture (Fig. 19) is the $M$-word shift register. The solution allowing minimization of the overall test hardware 


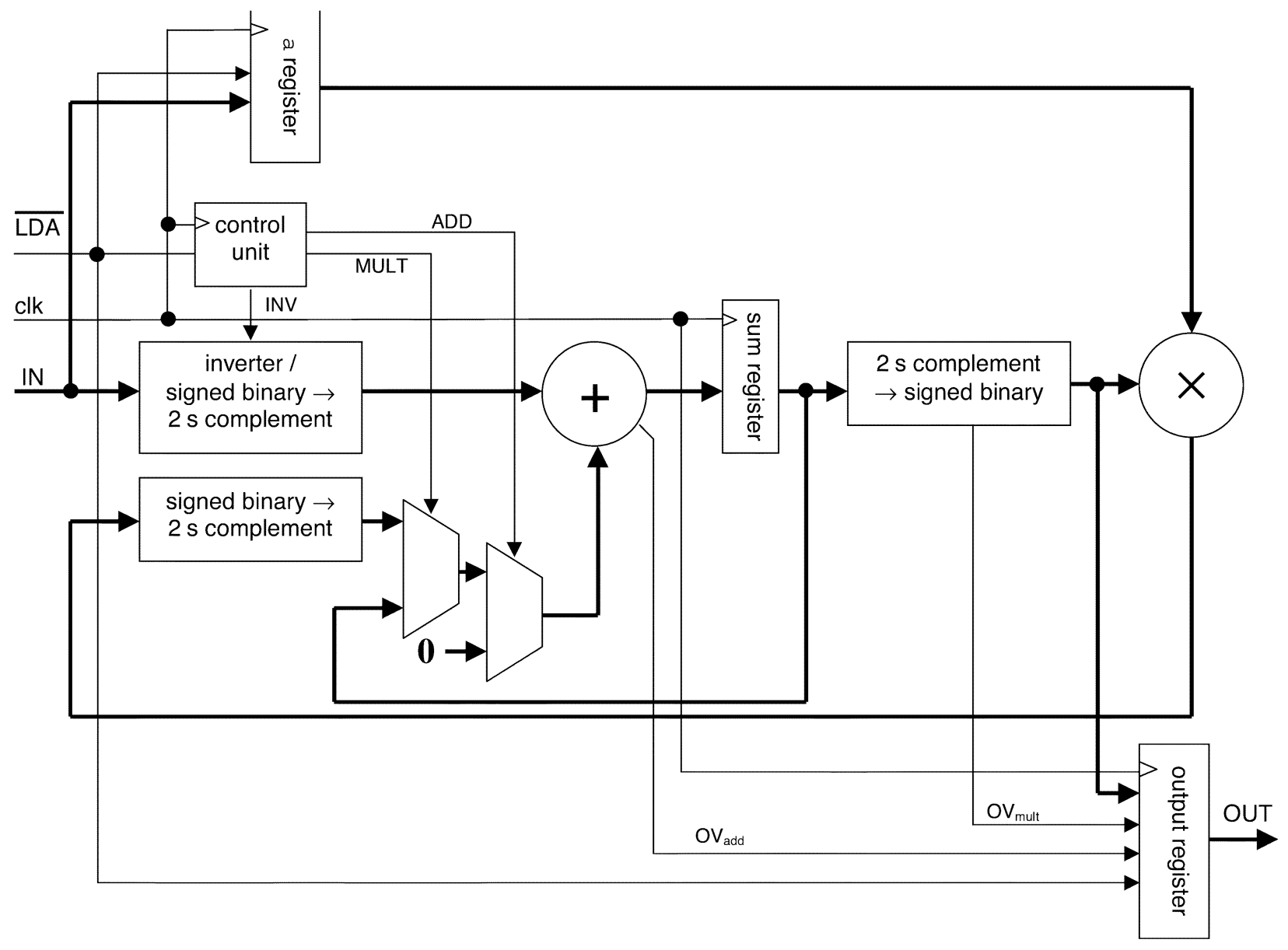

Fig. 20. SW ALU data flow.

overhead could include employing the same register for pseudorandom test sequence generation (that is normally based on a linear feedback shift register [6]). In addition, significant hardware savings can be achieved by using memory elements of the Boundary Scan (IEEE Std. 1149.1 and later standards [5], [6]) chip peripheral register to implement the SW network (if the DUT complies with the Boundary Scan design architecture and rules).

\section{APPENDIX I}

Discrete convolution between two sequences $\left\{f_{n}\right\}$ and $\left\{g_{n}\right\}$ is defined as

$$
f_{n} * g_{n}=\sum_{k=-\infty}^{\infty} f_{k} g_{n-k}
$$

Define a mapping, $\psi_{k, n}$, between $\left\{f_{n}\right\}$ and $\left\{g_{n}\right\}$ as

$$
f_{n}=\sum_{k=-\infty}^{+\infty} g_{k} \psi_{k, n}
$$

If $\left\{g_{n}\right\}$ is the convolution of two sequences,

$$
g_{n}=g_{1, n} * g_{2, n}
$$

then the mapping preserves convolution only if

$$
f_{n}=f_{1, n} * f_{2, n}
$$

where $\left\{f_{1, n}\right\}$ and $\left\{f_{2, n}\right\}$ are the respective mappings of $\left\{g_{1, n}\right\}$ and $\left\{g_{2, n}\right\}$ according to (34).

If $F[z], F_{1}[z]$, and $F_{2}[z]$ are the $z$-transforms of $\left\{f_{n}\right\},\left\{f_{1, n}\right\}$, and $\left\{f_{2, n}\right\}$ respectively. In the $z$ domain, convolution reduces to multiplication, so taking the $z$-transform of (36) gives:

$$
F[z]=F_{1}[z] \cdot F_{2}[z] .
$$

If we also define $\Psi_{k}[z]$ to be the $z$-transform of $\psi_{k, n}$, then (34) can be expressed in the $z$ domain as

$$
F[z]=\sum_{k=-\infty}^{+\infty} g_{k} \Psi_{k}[z] .
$$

Substituting this into (37) gives:

$$
\sum_{n=-\infty}^{+\infty} g_{n} \Psi_{n}[z]=\sum_{k=-\infty}^{+\infty} g_{1, k} \Psi_{k}[z] \cdot \sum_{n=-\infty}^{+\infty} g_{2, n} \Psi_{n}[z]
$$


Now if we substitute (35) in the left hand side, and combine the summations on the right hand side we get

$$
\begin{aligned}
& \sum_{n=-\infty}^{+\infty} \sum_{k=-\infty}^{+\infty} g_{1, k} g_{2, n-k} \Psi_{k}[z] \\
& =\sum_{n=-\infty}^{+\infty} \sum_{k=-\infty}^{+\infty} g_{1, k} g_{2, n-k} \Psi_{k}[z] \Psi_{n-k}[z]
\end{aligned}
$$

For (40) to be true for all $\left\{g_{1, n}\right\}$ and $\left\{g_{2, n}\right\}$ it must hold that

$$
\Psi_{k}[z]=\Psi_{k-n}[z] \Psi_{n}[z]
$$

which is equivalent to

$$
\Psi_{k}[z] \Psi_{n}[z]=\Psi_{k+n}[z]
$$

For $n=1$ we get the following difference equation:

$$
\Psi_{k+1}[z]-\Psi_{1}[z] \Psi_{k}[z]=0
$$

which has as its only nontrivial solution

$$
\Psi_{k}[z]=\left(\Psi_{1}[z]\right)^{k}
$$

\section{APPENDIX II}

\section{High Frequency Emphasis Filter PSPICE Netlist}

- high frequency emphasis filter

- power supplies

V1 $50 \mathrm{dc}+12 \mathrm{~V}$

V2 $60 \mathrm{dc}-12 \mathrm{~V}$

- signal input

Vin $10 \mathrm{AC} 10$

- filter circuit

R1 $021.2 \mathrm{k}$

C1 2333 n

R2 $341.2 \mathrm{k}$

Rload $4010 \mathrm{Meg}$

XU 113564 LM324

- LM324 op-amp subcircuit

- connections: noninverting input

- | inverting input

- || positive power supply

- || negative power supply

- |||| output

- |||| $\mid$

.subckt LM324 12345

...〈op - ampsubcircuit $\rangle \ldots$

.ends

\section{ACKNOWLEDGMENT}

The authors would like to thank C. Low of Singapore Polytechnic and E. Sicard of INSA, France for their assistance in the spectral warping ALU chip design. The authors are also very grateful to the Asia 2000 Foundation, Royal Society of New Zealand and the Embassy of France in New Zealand for their supporting the collaboration links between the authors, as well as with their overseas partners involved in the research presented in the paper.

\section{REFERENCES}

[1] M. Soma, "Mixed-signal fault models and design for test (I)," in Proc. IEEE Asian Test Symp., Nov. 1996.

[2] C. -L. Wey, "Mixed-signal fault models and design for test (II)," in Proc. IEEE Asian Test Symp., Nov. 1996

[3] "Special issue on analog and mixed signal testing," J. Electronic Testing: Theory and Applications (JETTA), vol. 9, no. 1/2, 1996.

[4] "Special issue on the IEEE international mixed signal testing workshop (IMSTW)," J. Electronic Testing: Theory and Applications (JETTA), vol. 17, no. 5, 2001.

[5] A. Osserian, Ed., "Analog and mixed-signal boundary-scan: A guide to the IEEE 1149.4 test standard," in Frontiers in Electronic Testing. Boston: Kluwer Academic Publishers, 1999, vol. 16.

[6] M. Bushnell and V. Agarwal, "Essentials of electronic testing for digital, memory, and mixed-signal VLSI circuits," in Frontiers in Electronic Testing. Boston: Kluwer Academic Publishers, 2000, vol. 17.

[7] G. W. Roberts and A. K. Lu, Analog Signal Generation for Built-In Self-Test of Mixed-Signal Integrated Circuits. Dordrecht: Kluwer Academic Publishers, 1995.

[8] M. Mahoney, DSP-Based Testing of Analog and Mixed-Signal Circuits: IEEE Computer Society Press, 1987.

[9] C. Marven and G. Ewers, A Simple Approach to Digital Signal Processing. NY: John Wiley, 1996.

[10] A. V. Oppenheim, D. H. Johnson, and K. Steiglitz, "Computation of spectra with unequal resolution using the fast fourier transform," Proc. IEEE, vol. 59, pp. 299-301, Feb. 1971.

[11] A. V. Oppenheim and D. H. Johnson, "Discrete representation of signals," Proc. IEEE, vol. 60, pp. 681-691, June 1972.

[12] S. N. Demidenko and K. Lever, "Analysis of the frequency characteristics of a digital spectral warping network," in Proc. Int. Workshop on Design Methodologies for Microelectronics and Signal Processing, Gliwice-Cracow, Poland, 1993, pp. 397-401.

[13] J. Holub, R. Šmíd, and J. Vedral, "A new time-saving method for ADC testing," in Proc. Measurement '99 Conf., Bratislava, Slovak Republic, 1999 , pp. $55-58$

[14] — "Chirp based method for ADC testing: Simulation and evaluation," in Proc. Conf. Advanced A/D and D/A Conversion Techniques and Their Applications., Glasgow, UK, 1999, pp. 94-96.

[15] IEEE Standard Terminology and Test Methods for Analog-to-Digital Converters, IEEE Std 1241-2000, 2000.

[16] S. K. Mitra, Digital Signal Processing: A Computer-Based Approach, Singapore: McGraw-Hill, 1998, pp. 443-445.

[17] W. Allen, D. Bailey, S. Demidenko, and V. Piuri, "Analysis and implementation of a spectral warping network," in Proc. Int. Symp. Integrated Circuits, 2001, pp. 492-496.

Warwick P. M. Allen received the B.E. degree in Information Engineering in 2003 from Massey University, Palmerston North, New Zealand. From April 2003 to April 2004, he worked at Singapore Polytechnic, designing and fabricating micro-electromechanical systems (MEMS). Mr. Allen returned to Massey University in 2004 to undertake postgraduate study. Mr. Allen also lectures in computer science at Massey University.

Donald G. Bailey (M'80) received the B.E. (Hons) degree in Electrical Engineering in 1982, and the Ph.D. degree in Electrical and Electronic Engineering from the University of Canterbury, New Zealand. From July 1985 to March 1987, he applied image analysis to the wool and paper industries at Wool Research Organization of New Zealand and Pulp and Paper Research Organization of New Zealand respectively as a visiting scientist. From April 1987 to October 1989 he was a visiting research engineer at University of California at Santa Barbara. Dr. Bailey joined Massey University in Palmerston North New Zealand as Director of the Image Analysis Unit. He is currently a Senior Lecturer in the Institute of Information Sciences and Technology at Massey University. 
Serge N. Demidenko received his M.Eng. in Computer Engineering in 1977 from the Belarusian State University of Informatics and Radio Electronics, the $\mathrm{Ph} . \mathrm{D}$. in Engineering Cybernetics and Information Theory in 1984 from the Institute of Engineering Cybernetics of the Belarusian Academy of Sciences, Associate Professor by Research (Senior Scientist) Academic Degree in Control Engineering in 1990 from the Academy of Sciences of the USSR, and Associate Professor by Teaching (Docent) Academic Degree in 1998 from the Belarusian State University of Informatics and Radio Electronics. During his career in the country he progressed from an engineer position to Chief of Join (Industry-Academy) Test Laboratory of a big manufacturing enterprise (around 12000 staff), and Head of Department posts working for academia and industry. Besides this, he worked also as academic staff for several institutions of higher learning. Currently Dr. Demidenko is a Chair of Electrical and Computer Systems Engineering of Monash University, Malaysia Campus.

His research interests include design and test of digital circuits and systems, testability, fault-tolerance, digital signal processing and generation. The list of his publications includes 4 books, more than 80 papers and 25 patents.

Serge Demidenko is a Senior Member of IEEE, Fellow and International Council Representative of IEE, and UK Chartered Engineer. Since 1998 he is a Vice-Chair of Asia-Pacilc activities of the Test Technology Technical Counci under the IEEE Computer Society, and a Co-Chair of Technical Committee on Fault Tolerant Measurement Systems under IEEE Instrumentation and Measurement Society. He was serving on the program and organizing committees of many international conferences and workshops. He is currently on the editoria board of the "JETTA: Journal of Electronic Testing: Theory and Applications" and IEEE TRANSACTIONS ON INSTRUMENTATION AND MEASUREMENT.
Vincenzo Piuri obtained the Ph.D. in Computer Engineering in 1989, at Politecnico di Milano, Italy. From 1992 to September 2000, he was Associate Professor in Operating Systems at Politecnico di Milano. Since October 2000 he is Ful Professor in Computer Engineering at the University of Milano, Italy. He was Visiting Professor at the University of Texas at Austin during the summers from 1993 to 1999.

His research interests include distributed and parallel computing systems, computer arithmetic, application-specific processing architectures, digital signal processing architectures, fault tolerance, neural network architectures, theory and industrial applications of neural techniques for identification, prediction, control, signal and image processing. Original results have been published in more than 150 papers in book chapters, international journals, and proceedings of international conferences.

$\mathrm{He}$ is a Fellow Member of the IEEE and member of ACM, IMACS, INNS, AEI. He is Associate Editor of the IEEE TRANSACTIONS ON NEURAL NeTWORKS, the IEEE TRANSACTIONS ON INSTRUMENTATION AND MEASUREMENT, and the Journal of Systems Architecture. He is Vice President for Publications of the IEEE Instrumentation and Measurement Society, Vice President for Members Activities of the IEEE Neural Networks Society, and Member of the Administrative Committee both of the IEEE Instrumentation and Measurement Society and the IEEE Neural Network Society. He is Chair of the Technical Activity Committee on Defect Tolerance of the Test Technology Technical Council of the IEEE Computer Society. 


\title{
Analysis and application of digital spectral warping in analog and mixed-signal testing
}

\author{
Allen, W. R. M. \\ 2003-12 \\ http://hdl.handle.net/10179/9640 \\ 22/04/2023 - Downloaded from MASSEY RESEARCH ONLINE
}

